\title{
A Lean Manufacturing Initiative to Streamline the Advising Process
}

\section{Dr. Corey Kiassat P.E., Quinnipiac University}

Dr. Corey Kiassat is an Associate Professor of Industrial Engineering, and the Associate Dean of the School of Engineering at Quinnipiac University. He has a BASc and a PhD degree in Industrial Engineering from the University of Toronto, and an MBA, majoring in Marketing and International Business, from York University. Corey is a Professional Engineer and has 11 years of industry experience in manufacturing engineering and operations management with General Motors in USA and Canada. He has also been involved with a start-up company in personalized preventive healthcare. Corey's research focus is on the role of people on performance of systems. His general research interests include process optimization in the healthcare industry.

\section{Prof. Ruby Elkharboutly, Quinnipiac University}

Prof. Ruby ElKharboutly an Associate Professor of Software Engineering at Quinnipiac University. She has a $\mathrm{PhD}$ in Computer Science and Engineering from University of Connecticut and a MS in Computer Science from the American University in Cairo. She has over twelve years of experience teaching in public and private institutions. Here research focuses on social data analysis and modeling. Her general research includes pedagogical research that enhances students class experience and interdisciplinary research in which software engineering is used to empower other disciplines. 


\title{
Lean Manufacturing Initiative to Streamline the Advising Process, A Work-in-progress
}

\author{
Abstract \\ In this work-in-progress, we describe a novel advising process that employs student Peer \\ Mentors (PM) to enhance the operational efficiency of academic advisors. A "Lean \\ manufacturing" framework is used to classify all activities as value-added or non-value- \\ added ones. By applying this framework to our current advising process for first-year \\ students within the School of Engineering at Quinnipiac University, only activities requiring \\ faculty expertise, such as guidance and mentorship, are considered value-added and are \\ performed by the faculty advisor. There are other frequently-performed advising activities, \\ such as navigating the course management system, that do not require the expertise of a \\ faculty member. These tasks are deemed to be non-value-added tasks when defining value \\ from a faculty's perspective. As such, these tasks are handled by the PM, a senior in the \\ major. The PM meets with the student for a pre-advising session. S/he compiles an executive \\ summary of this pre-advising session and provides it to the faculty advisor prior to the \\ faculty advising session with the student. This framework is analogous to healthcare \\ environments where a nurse meets with a patient first inquire on the patient's symptoms and \\ to measure the vitals. The notes are then passed on to the medical provider as a high-level \\ summary in order to save the medical provider's time.
}

After running a first semester pilot of this ongoing study, the proposed process has resulted in shorter and more streamlined advising sessions. It has also resulted in higher advisor and student satisfaction.

\section{Introduction}

First-year student advising is critical to student's retention and path to success [1]. Significant work has been done to enhance the advising process in multiple universities [2]. Northwestern University’s McCormick School of Engineering and Applied Science developed a new advising process for first-year students by employing advisors that are also First-Year Seminar teachers. Peer advisors are also part of the model. They are responsible 
for guiding the students to university resources, and help them normalize their experiences through social events and group discussions [3]. The College of Engineering at Rowan University report their enhanced dual advising program in [4] . In this model, students are assigned a first-year advisor who is responsible for course sequencing, college and campus policies and procedures, and referral to campus resources such as tutoring, study abroad, while the faulty advisor provides discipline-specific academic, industry and career related guidance.

Advising is particularly important for engineering students. The results of a study performed on 113 undergraduates who left Engineering in 2004, 2007 and 2008 show that the top three factors are: poor teaching and advising, the difficulty of the engineering discipline, and a lack of "belonging" within engineering [5].

Our project framework is based on Lean Manufacturing principles [6]. Originally derived from the Toyota Production System. Lean is a philosophy that aims to improve the efficiency of a system by eliminating waste and continuous improvement [7]. While the origins of Lean hail from the manufacturing industry, service industries, such as healthcare, have adopted Lean and realized much success [8] [9].

The main goal of this study is to enhance operational efficiency of faculty advisors across the School of Engineering at Quinnipiac University, through a streamlined advising process that creates value and eliminates waste. Applications of Lean manufacturing concepts in academia are limited but not unprecedented [10] [11]. The proposed process in this paper provides the faculty with efficient and productive advising sessions. Tasks within the advising process deemed to be value-added are identified to capture best practices and to standardize across the faculty. Tasks deemed to be non-value-added as indicated by faculty are passed on to the PMs. While we postulate a secondary advantage of the PM model also helping student retention and success rate, we do not cover this advantage in the paper.

After this introductory section, the discussions in this paper follow the steps described in Figure 1. The initial data collection, leading to setting the baseline and designing the advising process are described first, before a short discussion on the pilot study. Next, we discuss the 
full implementation of the proposed advising process and the analysis of the results. Lastly, we conclude the paper and discuss future work.

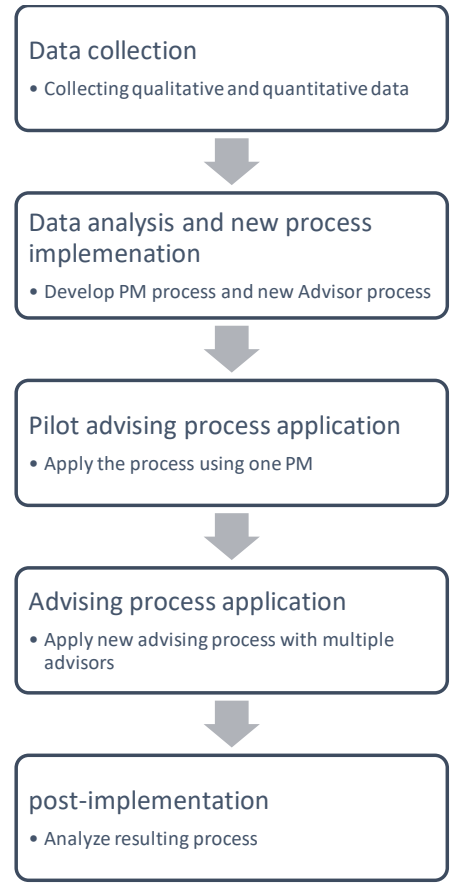

Figure 1 Study Framework

\section{Method}

The proposed advising process focuses on value creation and elimination of waste. As we pursue this goal, the mean duration, as well as the standard deviation, of faculty advising sessions is reduced, without jeopardizing the quality of the advising sessions. Therefore, we hypothesize the following: $\mu_{1}<\mu_{2}$ and $\delta_{1}<\delta_{2}$, where $\left\{\mu_{1}, \delta_{1}\right\}$ and $\left\{\mu_{2}, \delta_{2}\right\}$ are the mean and standard deviation of the proposed and current process, respectively.

Advising sessions across multiple faculty members are analyzed and broken down into a set of tasks. An ideal advising process is devised, capturing the best practices, as well as eliminating and/or reducing non-value-added tasks. Within the context of an advising session, "value" is defined as students receiving mentorship and assistance in achieving academic and intellectual growth. Faculty time directly related to such mentorship efforts is considered value-added. Examples include discussion on minors complementary to the major or participation in the Grand Challenge Scholar Program. Any faculty time not directly related to mentorship efforts is considered non-value-added. Examples include showing the 
students how to register for courses on Self Service, the course management system, or where to find the list of Humanities courses, a subset of which are graduation requirements.

The overall duration and variance of advising sessions is reduced through two general categories of effort. The first focuses on value-added activities, with the goal of capturing the best practices across the faculty. To demonstrate consider faculty members A and B who discuss the benefit of having a Mathematics minor with their advisees. Faculty member A holds a discussion for 2 minutes on average, while B spends 4.5 minutes on average. If the essential points covered are the same, then A's talking points can be captured as best practice and passed on to Faculty B in order to reduce B's duration by 2.5 minutes.

The second effort is focused on finding tasks traditionally performed during advising sessions that do not require the expertise of a faculty member with a $\mathrm{PhD}$. While first-year students are provided all the necessary information at orientation, they encounter an information overload and fail to remember certain procedures. How to register for courses on Blackboard, where to find the list of courses within the general university curriculum (humanities, Social Science, and Fine Arts), and who to approach about issues at their resident halls, are examples of tasks the students need help with but the help does not necessarily need to come from a faculty member. Such tasks considered as non-value-added tasks and are passed on to the PM.

At the onset of the project in Fall 2017, six faculty members volunteered to participated in the study. These six faculty members had a range of six to 22 first-year student advisees, for a total of 59 first-year student advisees across them all. Their participation required capturing the total duration of each advising session and participating in a post-advising interview. In addition, they were asked to follow a suggested advising script prior as much as possible. The advising script, design by the authors, attempted to break down the advising session into a set of tasks and provided a first draft of a standardized advising process. Through a postadvising interview, we were able to extract the following three factors: 1) what advising tasks are commonly performed that can be eliminated, 2) are there any best practices from one (or a few) individuals that can be captured to streamline the value-added tasks, and 3) which tasks can be passed on from faculty members to PMs. 
At the end of Fall 2017 term, the faculty advising script was finetuned. Moreover, a PM advising script including a list of tasks was created. A pilot study was run in the Spring 2018 term with one faculty advisor, who is a member of the research team, and one PM. The faculty advisor had 10 first-year student advisees. While Spring advising differs from Fall advising mainly due to the first-year students being more knowledgeable, there was still an opportunity to further finetune the proposed process. In addition, the PM selected for the pilot study, a junior student at this time, gained the necessary experience to act as the lead PM during Fall 2018 advising period.

In Fall 2018, a full implementation of the proposed advising model took place for the majority of first-year students. Six PMs were trained early in the Fall term. The research team explained the goal of the project to the PMs and emphasized the dual role the PMs can play in streamlining the advising sessions of the faculty members, as well as being a mentor and passing on their experiences to students' advisors. Furthermore, the PMs were handed an advising script, sample executive summaries, collected from the pilot in the Spring term and a list of first-year student advisees assigned to each PM and their faculty advisors. First-year students were informed about the PM as a great resource and the role the PMs were expected to play in advising. The PMs duties are to hold one-on-one meetings with their advisees, follow the proposed advising script, and provide an executive summary to the assigned faculty member after the pre-advising sessions.

The proposed faculty advising script was shared with 13 faculty advisors who were informed about the role the PMs were expected to play. Each faculty member would read the executive summary of their advisee before the advising meeting with the particular student. The research team collected session durations from the faculty who had participated in the study in Fall 2017. These durations are used to calculate the mean and standard deviation of the new process, compared to the baseline of Fall 2017. All 13 faculty were to complete a postadvising survey. 


\section{Results and Discussion}

\subsection{Duration and Variation of Advising Sessions}

Table 1 displays the number of advising sessions for each advisor and the calculated mean and standard deviation of advising sessions in Fall 2017 when the baseline was established., The overall mean and standard deviation across six faculty and 59 students were 30.4 and 9.3 minutes, respectively. Among the faculty, mean and standard deviations ranged from 22.7 to 39.3 , and 4.1 to 9.8 , respectively.

\begin{tabular}{|c|c|c|c|}
\hline Advisor \# & No. of advisees & Mean & Std. deviation \\
\hline $\mathbf{1}$ & 9 & 39.3 & 6.2 \\
\hline $\mathbf{2}$ & 22 & 31.4 & 8.5 \\
\hline $\mathbf{3}$ & 6 & 25.7 & 5.2 \\
\hline $\mathbf{4}$ & 6 & 22.7 & 4.1 \\
\hline $\mathbf{5}$ & 6 & 34.0 & 6.5 \\
\hline $\mathbf{6}$ & 8 & 29.8 & 9.8 \\
\hline Overall & 59 & 30.4 & 9.3 \\
\hline
\end{tabular}

Table 1 Mean and standard deviation of advising sessions

Table 2 shows the number of advisees, mean and standard deviation of advising sessions using the new process in Fall 2018. Unfortunately, three of the six original faculty were excluded as they had no first-year student advisees this year. The overall mean and standard deviation across three faculty and 25 students were 27.5 and 7.5 minutes, respectively. Among the faculty, mean and standard deviations ranged from 23.2 to 33.4 , and 5.3 to 7.0, respectively.

\begin{tabular}{|c|c|c|c|}
\hline Advisor \# & No. of advisees & Mean & Std. deviation \\
\hline $\mathbf{1}$ & 10 & 23.2 & 5.3 \\
\hline $\mathbf{2}$ & 8 & 33.4 & 6.1 \\
\hline $\mathbf{3}$ & 7 & 25.7 & 7.0 \\
\hline Overall & 25 & 27.5 & 7.5 \\
\hline
\end{tabular}

Table 2 Number of advisees, mean and standard deviation of advising sessions using the new process

At first glance, it is easy to see a reduction of duration and variation for the overall process. Referring back to the hypothesis stated in Section $2,\left\{\mu_{1}=27.5, \delta_{1}=7.5\right\}$ and $\left\{\mu_{2}=30.4, \delta_{2}=9.3\right\}$, we can see that neither inequality of $\mu_{1}<\mu_{2}$ or $\delta_{1}<\delta_{2}$ is rejected. 
However, this between-subject analysis needs to be revisited and statistically confirmed once a larger sample size is available in Fall 2019.

The results look positive for the within-subject analysis of our proposed advising process. For the baseline, the mean and standard deviations ranges were (22.7-39.3), and (4.1-9.8), respectively. For the proposed process implemented in Fall 2018, the mean and standard deviations ranges were (23.2-33.4), and (5.3-7.0), respectively. Similar to the betweensubject analysis, we need to have a larger sample size after Fall 2019 before we can state that the within-subject results have a significant statistical improvement.

\subsection{Qualitative Results from Faculty Surveys}

A post-advising survey was administered to all 13 faculty whose 91 advisees had meetings with the PMs. The survey included three questions and a comments section as shown in Figure 2 Post-advising survey.

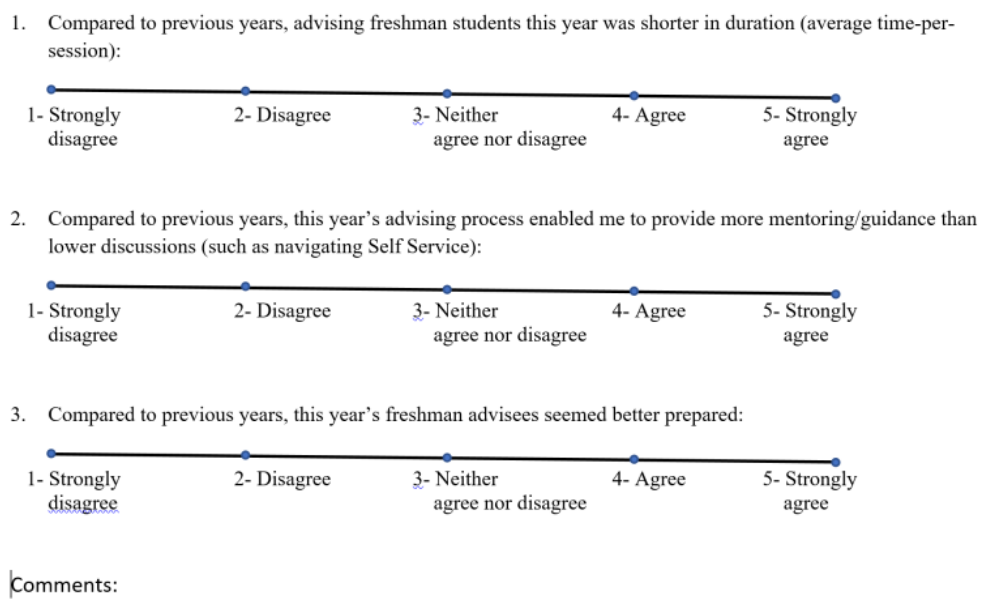

Figure 2 Post-advising survey

Nine of the 13 faculty responded to the survey. For question 1, the post-survey results indicate an average score of 3.33 out of 5 , indicating the faculty slightly agree that the advising process is shorter this year compared to the previous years. The average score for question 2 is 3.78 out of 5, indicating that the faculty agreed this year's advising process was more impactful and efficient. Finally, for question 3, the average score was once again 3.78 out of 5. The faculty agree that compared to previous years, their first-year student advisees 
are better prepared as a result of meeting with a PM. All three of the average scores qualitatively support the new advising process.

\section{Conclusion and Future work}

This paper describes a new advising process for first-year students in the School of Engineering at Quinnipiac University, based on a Lean Manufacturing framework. The purpose of this process is to enhance the operational efficiency across the faculty by streamlining the faculty's advising sessions. This approach follows the Lean/Six Sigma model through identifying value-added activities versus non-value-added activities within a process to reduce wasted resources and optimize a process. Non-value-added activities are offloaded to PMs in the new advising process. The proposed process has been applied during Spring 2017 on trial bases and was fully implemented during the Fall 2018 advising season. Results show a significant reduction in the advising session duration and general satisfaction from both faculty and students.

The study is still ongoing. The next steps involve standardization and validation. We plan to standardize the process by reflecting and rectifying problems that aroused during Fall 2018. Some problems included miscommunication between PMs and faculty and delays in obtaining the executive summaries. We would like to expand the use of the new advising process to including all advising faulty and all first-year students in Fall 2019.

The validation/post-implementation phase is conducted through and further analyzing data to test the results of between-subject and within-subject analyses of mean and standard deviation of the advising sessions using a larger sample set. Retention data will be analyzed as well to validate the hypothesis that our proposed process would yield higher retention rates for first-year students.

\section{Acknowledgements}

The authors would like to thank Provost Thompson for providing us with an Innovation Grant to pursue this endeavor. The authors would like to thank Andrew Koukoulas, the Lead Peer Mentor, who helped us during the pilot stage, as well as the launch in the academic year. 
finally, the authors would like to thank Professors Christov, Fonseca, Kile, Reap, and Riofrio who volunteered to help during the launch of the Peer Mentor program.

\section{References}

[1] H. Swecker, M. Fifolt and L. Searby, "Academic Advising and First-Generation College Students: A," NACADA Journal, vol. 33, no. 1, pp. 46-53, 2013.

[2] K. N. Tuttle, Academic Advising, New Directions for Higher Education, 2000.

[3] R. W. Freeman, K. Gentry and J. E. Goldberg, "Changing the Advising Model," in 2016 ASEE Annual Conference \& Exposition, 2016.

[4] J. W. Everett and M. Perez-Colon, "Evaluation of a dual first year student advising program," in 122nd ASEE Annual Conference and Exposition, Seattle, MA, 2015.

[5] R. M. Marra, K. A. Rodgers, D. Shen and B. Bogue, "Leaving Engineering: A MultiYear Single Institution Study," Journal of Engineering Education, vol. 101, pp. 6$27,2012$.

[6] J. F. Krafcik, "Triumph of the Lean Production System," Sloan Management Review, vol. 30 , no. 1 , pp. 41-52, 1988.

[7] J. P. Womack, D. T. Jones and D. Roods, The Machine that Changed the World, New York: Rawson Associates Scribner, 1990.

[8] R. W. Bush, "'Reducing Waste in US Health Care Systems"," Journal of American Medical Association, vol. 297, no. 8, pp. 871-874, 2007.

[9] R. W. Simon and E. G. Canacari, "A Practical Guide to Applying Lean Tools and Management Principles to Health Care Improvement Projects," Association of Perioperative Registered Nurses Journal, vol. 95, no. 1, pp. 85-103, 2012. 
[10] A. N. Nambiar and A. Dutta, "Expert system for student advising using JESS," in 2010 International Conference on Educational and Information Technology, Chongqing, 2010.

[11] W. W. Fisher, S. Barman and P. L. Killingsworth, "Value stream mapping for improving academic advising," International Journal of Information and Operations Management Education, vol. 4, no. 1, pp. 45-49, 2011. 